Received: November21,2016

Accepted: December30,2016

Published: January10,2017

\section{Autism and B-Vitamin Deficiency Linked to Alcohol and Sugar Intake and the WIC Program}

\author{
Raymond J Shamberger* \\ Emeritus Laboratory Director, King James Medical Laboratory, Kirtland
}

*Corresponding author: Raymond J Shamberger, FACN, Emeritus Laboratory Director, King James Medical Laboratory, Kirtland, Ohio 44094, Tel: 440-256-1223; E-mail: Bobray @ameritech.net

\section{Abstract}

Objective:The CDC has warned against drinking while pregnant noting in guidelines this year that no amount of alcohol is advised. The CDC has also previously recommended abstention in regard to Fetal Alcohol Syndrome (FAS) whose alcohol intake varies with the amount of binge drinking. The objective is to compare autism rate to alcohol consumption.

Method:The Institute for Health Metrics at Seattle Washington has listed the average alcohol consumption for 48 states. For 2009 the autism rates are known and the average alcohol consumption are listed. In addition, the alcohol rate is also known for all counties. These results were compared to previously reported WIC rates and autism rates that had been previously reported in the counties of three states.

Results:Comparison of the autism rate to the alcohol consumption rate showed a strong positive relationship. In the counties of three states there was also a strong direct relationship of alcohol consumption to the autism rate. These counties showed an inverse relationship to the WIC rates.

Conclusions:The results of a positive correlation between alcohol consumption and the autism by states and counties is consistent with the precautions cited by the CDC.

\section{Keywords:}

Autism; Binge Drinking; WIC; Alcohol; Fetal Alcohol and Thiamine;

\section{Introduction}

The rate of autism is increasing 10-15\% per year. The autism spectral disorder is a group of developmental disabilities that can cause significant social communication and behavioral challenges. The cause of autism is unknown. Autism usually appears in children ages 2-4. The child may have no speech or it might be severely delayed. The children may be socially awkward and ritualistic behavior may be followed. Some of these symptoms may appear by the time the baby is about nine months. Boys have a 4 to 1 chance of developing autism over girls. About 1.5 million children have some form of autism with 500,000 of these cases under age 21. The cases include several types of autism including Asperger's Syndrome, Rett's syndrome, disintegrative disorder and pervasive developmental disorder. Some postulated causes of autism include consumption of certain foods; infectious disease; heavy metals; solvents; diesel exhaust; phenol plastics; PCB's; pesticides; brominated flame retardants; glyphosate applied to corn and soy; alcohol; smoking; illicit drugs; rainfall; watching television and vaccines. The vaccine issue was of interest for awhile because parents became aware of autism around the time of inoculation. A mercury preservative, thimerosal, was suspected, but when the mercury was removed the autism rates seemed to become greater. In addition, there was no evidence that MMR-Measles, Mumps, and Rubella vaccines increased autism and the original vaccine study was discredited. Because of the wide-spread occurrence of autism and its increase in almost epidemic proportions, some more obvious unexpected causes may be responsible for the rapidly increasing rate of autism. The Center for Disease Control and Prevention warns against drinking while pregnant noting in guidelines this year that no amount of alcohol consumed is advised during pregnancy no matter how small. Many doctors recommend that women avoid alcohol for the entire nine months of pregnancy. The CDC has previously recommended similar abstention in regard to Fetal Alcohol Syndrome (FAS) whose concentration varies with the amount of binge drinking. The reason for possible increased damage is that the alcohol titer or level can arise to levels which can damage organs. Binge drinking is a dangerous form of alcohol consumption which is more prevalent in households with incomes of $\$ 75,000$. Binge drinking is a pattern of drinking that results in a blood alcohol concentration (BAC) to 0.08 gram per cent or more. In the United States [1] the overall prevalence of binge drinking was $17.1 \%$. The frequency of binge drinking was 4.4 episodes per month and the intensity was 7.9 drinks on occasion. The CDC 
analyzed data for women and found [2] 10\% of pregnant women used alcohol and about $2 \%$ engaged in in binge drinking or frequent alcohol Use. About 12.4\% reported some binge drinking.

\subsection{Excessive Alcohol Use}

Excess alcohol use, including binge drinking is responsible for 80,000 deaths annually in the United States. Excess drinking cost the American economy about \$ 223.5 billion in 2006. Binge drinking increases the risk of getting injured or injuring others. Excess drinking increases the risk of unplanned pregnancies and the spread of Sexually Transmitted Diseases (STD) due to unprotected sex. Most may stop drinking when they realize they are pregnant. Poor dietary habits as well as impaired absorption of nutrients from the blood stream due to alcohol-induced digestive changes may lead to vitamin deficiencies. A deficiency of folic acid can lead to anemia. In emergency room situations folic acid should be administered usually as a multivitamin for a few weeks following alcohol withdrawal. Also, thiamine $100 \mathrm{mg}$ daily should be given for a minimum of 3 days to all patients hospitalized with alcohol withdrawal. Thiamine, which plays a major role in energy metabolism is often deficient in people with alcohol dependence. If thiamine is continuously deficient, then a more serious deficiency of Wernike-Korsakoff syndrome may arise. This is characterized by paralysis of certain eye muscles, an abnormal gait, and severe confusion which might lead to irreversible dementia. Thiamine should be administered before glucose in any form is given in order to prevent the depletion of stored thiamine and the onset of Wernicke-Korsakoff syndrome. Sometimes patients are brought into the emergency room unconscious and cannot help with their treatment. If It is suspected that that are intoxicated they are not given glucose, but instead are administered a mixture of thiamine and folic acid. Glucose administration in these cases might be fatal.

\subsection{Alcohol Intake Problem and Autism}

The CDC has compared the average female age 18-44 binge drinking rate per state [3] to the autism rate of 8 year olds in that state. The results showed a direct relationship $\mathrm{r}=0.360, \mathrm{P}<0.05$. Comparisons of the United States total breast feeding per year [4] to the autism rate of 8 year olds for the years 2007 to 2010 showed a direct relationship to autism in the United States. Similar results have been observed for the years 2000-2004. The data suggests that some components of breast milk may be deficient in some nutrient or which may be affected by nutrition. The CDC (Communicable Disease Center) has stated that 1 in 6 American adults is a binge drinker.The problem is more widespread than thought, because alcohol drinking has been linked to car crashes, violence, STD's and death. The binge drinker does so an average of four times per month, sometimes downing eight drinks at one sitting. Binge drinking is defined as consuming four or more drinks on one occasion for a women and five or more drinks for a man. This pattern may bring the BAC level to $0.08 \%$ or more. The rate of binge drinking in the U.S. is the highest in the world. Despite the U.S. drinking age, binge drinking is the greatest problem for people under the age of 21 . About $50 \%$ of the alcohol is consumed in the United States by adults and about $90 \%$ by young people is consumed during a binge drinking session. European binge drinking levels reveal a different trend. In general, Europeans consume more alcohol than people on other continents. Drinking in moderation is an important part of European culture and social interaction. In these countries it is legal for teenagers older than 16 to enjoy alcohol in the privacy of their homes. Binge drinking is overwhelmingly absent from most European cultures. Britain is very high in binge drinking and may be the binge-drinking capitol of Europe. Britain's drinking pattern is different in that its drinkers consume alcohol less regularly than other European states. When they do drink they may drink the most at a single sitting. About $12 \%$ have reported that they have consumed up to ten drinks in a single night. English women who binge drink during the second trimester are more likely to have an underweight baby or a small gestational weight. [5] The babies born underweight have a greater risk of having neonatal complications, include breathing problems, respiratory infections, hypothermia and impaired neurodevelopment. In the United States women [6] showed a much faster escalation in binge drinking than men with rates rising 7.5\% between 2005 and 2012. In contrast men saw rates of binge drinking increase $4.9 \%$. Binge drinking is commonly associated with a higher risk of bodily harm such as alcohol poisoning and acute adult organ damage. The South Koreans are ahead of other ethnic groups in alcohol consumption and they have a very high rate of autism compared to the Americans. South Korean emigrants to the United States have a $24.6 \%$ incidence of binge drinking which is two or three times greater than other Asian countries [7]. In the urban areas of South Korea, about $46 \%$ of Korean men and about $9 \%$ of Korean women are heavy drinkers (more than 6 drinks per day). In contrast, only about 3.5\% of the United States population are heavy drinkers. In Korean Society they understood that drinking is an essential part of networking which is needed to succeed in the workplace. Many Koreans are first introduced to alcohol at universities and thereby develop unhealthy binge drinking habits. The Korean Alcohol Research Foundation in 2010 conducted a poll of 4061 students. More than $71 \%$ said they drank more than 5 shots of Soju in one sitting. This amount of alcohol consumption is defined as binge by WHO (World Health Organization). The large prevalence of binge drinking in South Korea may be associated with its high rate of autism with a prevalence of $2.6 \%$. In contrast, the American rate at the time was about $1 \%$. Fetal alcohol syndrome is also associated with binge drinking.

\subsection{Fetal Alcohol Syndrome}

Fetal alcohol syndrome refers to a group of physical behavioral and cognitive abnormalities. Children of binge drinking mothers have problems with distractibility, restlessness and lack of persistence. They also have the classic dysmorphic, facial features, prenatal and postnatal growth abnormalities and mental retardation. About $80 \%$ of affected children show poor coordination, hypotonic, attention-deficit hyperactivity disorder, decreased adipose tissue, facial abnormalities, maxillary, hypoplasia, cleft palate and micrognathia. Cardiac defects, hemangiomas and eye or ear ab- 
normalities are also common. Clinicians describe children with a variety of problems, including growth deficiency, behavioral mannerisms and delays in motor and speech performance. The Research Society on alcohol has defined it as any condition thought to be secondary to alcohol exposure in utero. Fetal alcohol syndrome usually occurs when a pregnant woman has more than four standard drinks per day. However, two drinks per day in the early part of pregnancy may result in milder symptoms. Among those who are alcoholic about a third of their children have FAS. Streisguth et al [8] have found children had abnormal cognitive functioning which manifests itself as poor attention and concentration skills, memory defects, impaired comprehension and abstract reasoning. Their IQ scores average around 70. FAS is estimated to occur at the rate of 5.2/1000 live births in the United States. Mills et al [9] have studied 31,000 pregnancies and the alcohol that women drank. Consumption of 1 or more drinks (a drink is defined as 1.5 ounce of distilled spirits, 5 ounces of wine and 12 ounces of beer was associated with an increased risk of having an infant with retardation. The potential damage to the fetus may cost the United States from 75 million to 9.7 billion. The total lifetime cost of caring for a typical child with FAS maybe as high as 1.4 million. Mental retardation related to FAS may account for as much as $11 \%$ of the annual cost of caring for all mentally institutionalized residents of the United States and about 5\% of all congenital abnormalities. The only way to prevent FAS is to avoid drinking alcohol during pregnancy. The Surgeon General [10] recommended in 1981, and again in 2005 that women abstain from alcohol use while pregnant or while planning a pregnancy. The latter way to avoid damage even in the earliest stages, even weeks, as the woman may not be aware she has conceived [11]. Mukherjee et al has also reported that drinking while pregnant can give babies FAS [12]. He compared autism as well as autistic traits in people exposed to heavy prenatal alcohol traits in a clinical series of 21 individuals in a nested case control study. The controls had not experienced prenatal alcohol exposure. The 21 with fetal alcohol spectrum disorders were assessed and 16 (72\%) met ICD-10 criteria for childhood autism.

\subsection{The WIC program}

The Special Supplemental Nutrition Program for Women, Infants and Children (WIC) is a federal assistance program of the Food and Nutrition Service (FNS) for low income families by the United States Department of Agriculture [13]. The eligibility requirement is between $100 \%$ but not more than $185 \%$ of the Federal Poverty Guideline. Effective from July 1, 2016 to June 30, 2017 the yearly allowance is $\$ 21,978$ for a family of 1 , with increases of $\$ 7,696$ for each additional member of the household. The program allows monthly purchases of healthful food stuffs. Purchase of alcoholic beverages is not subsidized by the program. The program is unrelated to the USDA's Supplemental Nutrition program (SNAP). Currently the WIC serves $53 \%$ of all infants born in the United States. Applicants must have a nutritional assessment by a qualified professional (physician, nurse, or nutritionist). The nutritional evaluation is based on height, weight, and growth assessment, hematocrit or hemoglobin levels, general health his- tory or a diet assessment, The applicants are given an EBT card which is exclusively used for purchasing WIC approved items. A wide variety of foods is allowed such as milk, breakfast cereal, cheese, eggs, fruits and vegetables. Some culturally appropriate food packages such as tortillas, brown rice canned salmon may also be provided. WIC has dramatically reduced health care costs by (a) providing prenatal services (b) promoting breast feeding. Several controlled evaluations showed that in 1992 prenatal WIC enrollment was estimated to have reduced first year medical costs for United States infants by $\$ 1.19$ billion, more than offsetting the government costs of WIC. Prenatal use of WIC also decreases the odds of having a low-weight newborn by 25 percent and reduces the very low birth-weight births by 44\%. Having a higher birthweight newborn has the potential to decrease costs of hospitalization in the first year. Infants with lower birth-weights have higher costs for initial hospitalization. Each dollar spent results in nearly three dollars saved by the federal and state governments. Because of these savings WIC is often cited as being one of the most costeffective food assistance programs in the nation [13]. In addition, WIC numbers are available for the county, states and all of the counties. Perhaps some relationship could be found between WIC nutrition and the autism rates. Although autism is thought to be caused by some types of mutations and not nutrition, perhaps some insight and direction could be found by looking at nutrition programs.

\subsection{The WIC program and Nutrition}

Because the autism rates were becoming known in some counties, states as well as the country, comparisons could be made to the WIC program to see if there could be a possible nutritional link. Autism is more likely to occur in families and mutagenic cause has been postulated. However, families also share the same food. Certainly, a nutritional cause of autism cannot be overlooked.Certainly a relationship could be explored between autism, nutrition and alcoholic consumption in the various states and counties.

\section{Statistical Analysis}

The Institute for Health Metrics at Seattle, Washington has studied the average drinking patterns in the United States. The year 2010 was selected. Data from two states Hawaii and Alaska were not available. The average drinking level in 48 states was compared to the 2009 autism rate using excel statistics. Trend lines were also compared visually to the alcohol consumption rates and the autism rates. In three states the county rates of WIC and autism were compared to the average alcohol intake of that county and to each other using excel statistics.

\section{Materials and Methods}

The Institute for Health Metrics at Seattle, Washington has studied the drinking patterns in US counties from 2002-2012 in 48 states (6). In $20126.2 \%$ of all Americans were considered heavy drinkers and $12.3 \%$ were considered binge drinkers. Two states Hawaii and Alaska were not included. There was considerable variance between the counties in each state. However, each state 
was given an average drinking level in that state. They divided the people of each county into three levels: level 1: no drinks; level 2; 1 drink per month and level 3: more than 1 drink per month. They compared no drinking to 1 or more drinks combined. The average drinking level per state was compared by correlation to the State 2009 autism rate[14] using excel statistics. The results are indicated in Figure 1 . The \% WIC recipients, and autism rates using Excel statistics in Washington, New Jersey, Oregon and in all 50 states or the counties of Washington, Oregon or New Jersey $[4,13]$ are listed in Table 1 . The counties that were used for the WIC study also had average alcohol intake for that county calculated from the Health Metrics Seattle study [6]. These results were also included in the Table 1 for comparison.

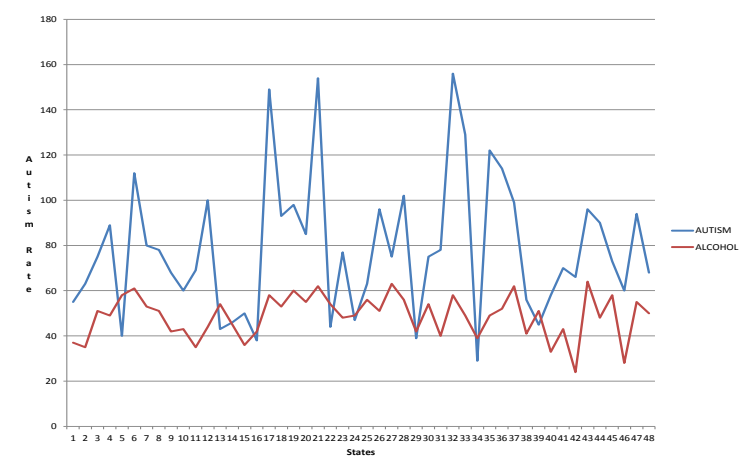

Figure 1: The autism rate and the average alcohol intake in 48 states in 2009.

Table 1: The WIC Program and the WIC/Autism and Alcohol/Autism Comparisons

\begin{tabular}{|c|c|c|c|c|c|c|}
\hline State & Year & Counties & WIC/Autism & $\mathrm{P}<$ & Alcohol/Autism & $\mathrm{p}<$ \\
\hline Washington & 2009 & 36 & -0.39 & $<0.02$ & +0.51 & $<0.02$ \\
\hline New Jersey & 2004 & 21 & -0.49 & $<0.05$ & +0.42 & $<0.05$ \\
\hline 0regon & 2010 & 30 & -0.39 & $<0.05$ & +0.38 & $<0.05$ \\
\hline All 50 states & 2004 & All & -0.37 & $<0.02$ & $-\ldots--$ & - \\
\hline
\end{tabular}

\section{Results and Discussion}

When the average drinking level by state was compared to the autism rate using Excel statistics by state for 2009 [6, 13], the result was $\mathrm{r} 0.52 \mathrm{p}<0.001$. There was also horizontal trend lines when the alcohol consumption were compared to the autism rates. Similar trend lines were found in 45 of 48 states. Even though the number of drinks was small compared to the final result In this study there was a large positive correlation to autism. The result may be compared with the CDC's caution of no alcohol during pregnancy. In the Table the counties of Washington, New Jersey and Oregon as well as all $50[4,13]$ states show an inverse relationship between the WIC infants and the rate of autism. In contrast WIC incidence to alcohol consumption in the same states showed that there was a direct relationship to alcohol consumption. Significantly, those on the WIC program had lower autism rates. Low-income mothers who qualify for the free milk formula from the WIC program seem to have an important source for their infants. In contrast, payment for alcoholic beverages is not supported by the WIC. Surgery items are also not supported by the WIC .Sugar is not a necessary nutrient for the body. The WIC families qualify because of lower income. However, individuals with higher income should be able to purchase more nutritious food and afford better health care and have a lesser incidence of diseases such as autism. It is well known that people with income over $\$ 75.000$ have a greater incidence of binge drinking which might explain higher autism rates in this population. Respondents with household income greater than $\$ 75,000$ had the highest binge drinking prevalence (20.2\%). This may explain why lower income people on WIC have less autism. Higher income people should be able to afford better health care.

\subsection{Autism Linked to Sugar Intake}

The USDA has measured the B-vitamin content of human breast milk from 279 mothers [16]. After calculating the average amount that the babies drink, comparisons can be made to the MDR (Minimal Daily Requirement) Because of the exclusive breast feeding that is recommended for the first six months it is possible that there could be a B-vitamin deficiency. In general, all of the B-vitamins were at acceptable levels except thiamine which was well below the MDR for the first four months. Part of this deficiency may be caused by the excess sugar intake in America. The historical increased sugar consumption from 1822 to2005 was examined for a possible connection to autism. In 1822 the per capita sugar consumption was about 8 lbs per person per year and by 2006 consumption reached over 100 lbs per year. Landon [17] constructed a graph from which a equation was derived, $\mathrm{y}=0.573 \mathrm{x} 1041$ where $\mathrm{x}=$ years. Total autism numbers can be calculated from the total national rate of autism from 2010 from the ADDM network (The Autism and Developmental Disabilities Network). These numbers are compared to the increasing rate of sugar consumption in the same years 2002-2012. Calculation of this relationship using excel statistics shows a formula result of $\mathrm{r}=0.976$ or $\mathrm{P}>0.001$. Thiamine is needed for the first step of the Krebs cycle and excess sugar demand could cause a thiamine deficiency. However excess alcohol intake may also contribute by decreasing thiamine absorption from the gastrointestinal tract. Children are more likely to develop autism if their mothers were diagnosed with diabetes early in pregnancy [18] in this study. Woman newly diagnosed by the 26th week of pregnancy were $42 \%$ more likely to have a child diagnosed with autism. The study included more than 322,000 children born between 1995 and 2009. However, mothers with type 2 diabetes, did not have children with increased rates of autism. This may have happened because the mothers may have already had their diabetes under control. The author of this study suggests that high blood sugar may have a lasting effect on a fetal organ development and brain function. Gardener et al [19] have studied prenatal risk factors for autism using a comprehensive meta-analysis. They also found 
that the maternal gestational diabetes was associated with a 2 fold increased risk of autism. The maternal age at birth if over 30 was associated with an increased risk of an autistic child. A five year increase in maternal age was associated with a $7 \%$ risk. Increased paternal age was also found to be a significant risk. The latter observation may not be as important because an older woman is probably married to an older man. Other studies have found an increased risk of autism for children who are born prematurely or very small. Genetics may be a risk factor if one child in a family has autism, the next child may have a $20 \%$ greater chance of having autism. In a large study [20] involving 1000 mother child pairs it showed that over a third of US women in their child bearing ages are obese and nearly one-tenth have gestational or type 2 diabetes during pregnancy. The percentage of children born to women with type 2 diabetes or gestational diabetes was $9.3 \%$. The developmental disability found was $11.6 \%$ higher than the $6.4 \%$ of children born to women without these metabolic conditions. Over $20 \%$ of the mothers of children with autism or other developmental disability were obese compared with $14 \%$ of the mothers of normally developing children. The authors concluded that the events of a poor regulated maternal glucose level and maternal inflammation may cause certain proteins involved in cell signaling to cross the placenta and adversely affect fetal development but that more studies are needed to verify this process.

\subsection{Does Sugar Directly Cause Autism?}

It would be very difficult to directly calculate autism from sugar intake because of the many types of sugar available for consumption. In addition, autism occurs in different rates in many states. However, we know the rates of sugar consumption have increased and we also know the rate of autism increase. The evidence linking high sugar and alcohol intake to increased autism was unexpected, however, both substances are known to decrease the amount of thiamine. In one case sugar reduces the absorption of thiamine into the intestine and alcohol directly reduces the absorption of thiamine by the intestine.

\section{Conclusion}

The advice given pregnant women to avoid alcohol is probably correct. The relationship between FAS and alcohol is fairly well established. In this study there is a substantial correlation between alcohol consumption and autism. In addition, the WIC results based on low alcohol intake had a negative correlation to autism. In contrast, the alcohol consumed in the counties of three states had a direct relationship to autism. This may mean that autism is directly enhanced by alcohol. The people in the counties on WIC had lower incomes and probably could not afford to binge drink whereas individuals with higher income could afford to binge drink. The mechanism by which binge drinking may be related to autism is unclear. However in another study [4] we showed that mother's milk was quite low in thiamin. In addition, alcohol lowers thiamine by decreasing thiamin absorption from the gut. High sugar intake can further decrease thiamine by reducing its concentration. Although evidence seems to point toward autism being a thiamine deficiency, more studies are needed to determine this possibility.

\section{References}

1. Morbidity and Mortality Weekly Report (MMWR). Vital Signs: Binge Drinking Prevalence, frequency and Intensity among adults in the United States. 2012;61(1):14-19.

2. Centers for Disease Control and Prevention (CDC). Alcohol consumption among women who are pregnant and who might become pregnant-United States 2002. Morbidity and Mortality Weekly Report. 2004;53(50):1178-1181.

3. CDC. Center for Disease Control and Prevention. State specific alcohol consumption for 2010 .

4. Shamberger R. Autism associated with B-vitamin deficiency linked to sugar intake and alcohol consumption. Journal of Intellectual Disability, Diagnosis and Treatment. 2015;3(1):7-12.

5. Cooper DL, Peterick ES. Wright. The association between binge drinking and birth outcomes. Results: Results from the born in Bradford cohorts. J Epidemiol Community Health. 2013;67(10):821-828. doi: 10.1136/jech-2012-202303.

6. Dwyer-Lingren L, Flaxman AD, Metal NG, Hansen GM, Murray CJ, Mokdad AH. Drinking patterns in US counties from 2002-2012. Amer J of Public Health. 2015;105(6):1120-1127. doi: 10.2105/AJPH.2014.302313.

7. Kim YS, Leverthal BL, KohJ L, Fombonne E, Laska E, Lim EC, et al. Prevalence of autism spectrum disorders in a total population sample. Am J Psychiatry. 2011;168(9):904-912. doi: 10.1176/appi.ajp.2011.10101532

8. Streisguth A, Barr H, Sampreon ID. Bookstein FL. Prenatal alcohol in offspring development the first 14 years. Drug Alcohol Depend.1994;36(2):89-99

9. Mills JL, Granbard BL, Harly EE, Rhoads GG, Berendes HW. Maternal alcohol consumption and birth weight. How much drinking in pregnancy is safe. JAMA. 1984;252(14):1875-1879.

10. Vice Admiral Richard H. Camona (A message to women from the U.S. Surgeon general on alcohol use in pregnancy). Washington, DC: US Department of Health and Human services.

11. Briggs, Gerald G. Drugs in pregnancy and lactation: A reference guide to fetal and neonatal risk. Philadelphia Wolters Kluver Health/ Lippincott Williams \& Wilkins. 2015.

12. Mukherjee R, Layton MY, Yacub E, Twoh J. Autism and autistic traits in people exposed to heavy prenatal alcohol: data from a series of 21 individuals and nested case control study. Advances in in mental health and intellectual disabilities. 
13. Aruch S, Puente CA. Savings achieved by giving WIC benefits to women prenatally. Public Health Reports. 1995;110(1):27-34.

14. Vaxtruth. When 1 in 88 is really 1 in 29 . Autism prevalence in public schools for 8 year olds in 2009-2010 school year. Vaxtruth org. 2009.

15. Shamberger RJ. Autism rates and the WIC program. J Am College of Nutrition. 2011;30(5):348-353

16. United States Department of Agriculture.

17. Paleodiabetic.com/tag/ Jeremy-Landen/ls a stone age diet healthy for diabetics. Jeremy-Landen. Posted on April 21, 2010.
18. Xiang AW. Wong X, Martinez MP, Walthall JC, Curry ES, Page $\mathrm{K}$, et al. Association of maternal diabetes with autism in offspring. JAMA. 2015;313(14):1425-1434. doi: 10.1001/jama.2015.2707.

19 Gardner H, Spiegalman D, Buka SL. Prenatal risk of factors for autism: Comprehensive meta-analysis. $\mathrm{Br} \mathrm{J}$ Psychiatry. 2009;195(1):7-14. doi: 10.1192/bjp.bp.108.051672.

20. Krakowiak, P Walker CK, Bremer AA, Alice S Baker, Sally Ozonoff, Robin L Hansen, et al. Maternal metabolic conditions and risk for autism and other neuro developmental disorders . Pediatrics. 2012;129(5):2011-2583 